Original Paper http://ajol.info/index.php/ijbcs http://indexmedicus.afro.who.int

\title{
Diversité floristique et dynamique de reconstitution de la forêt du Parc Zoologique sous plantations à eucalyptus à Brazzaville, Congo
}

\author{
Félix KOUBOUANA ${ }^{1 *}$, Suspense Averti IFO ${ }^{2}$, Lydie Blanche Mayitoukou LOUPET ${ }^{2}$ et \\ Eddie NDINGA ${ }^{2}$ \\ ${ }^{1}$ Laboratoire d'Ecologie Appliquée et Environnement, ENSAF, Université Marien Ngouabi, \\ Brazzaville, Congo. \\ ${ }^{2}$ Laboratoire d'Ecologie Appliquée et Environnement, ENS, Université Marien Ngouabi, Brazzaville, Congo. \\ *Auteur correspondant ; E-mail : koubouanafelix@gmail.com
}

\section{RESUME}

La forêt du Parc Zoologique est l'une des forêts naturelles reliques intra urbaines de la ville de Brazzaville. Cet écosystème subit une pression anthropique due aux besoins croissants en bois énergie, en bois d'artisanat et en plantes utilisées en phytothérapie. Les zones les plus dégradées ont été reboisées en eucalyptus. Il a été constaté que dans le sous bois des forêts à eucalyptus, se réalise la reconstitution de la forêt naturelle. Une étude diachronique a été effectuée pour suivre cette reconstitution. Des placettes de $600 \mathrm{~m}^{2}$ d'âges différents ont été délimitées dans ces plantations, dans lesquelles ont été notées toutes espèces de forêt naturelle existantes, leurs diamètres à la hauteur de la poitrine et leur nombre d'individus. Les résultats obtenus montrent que les types morphologiques des ligneux augmentent avec l'âge de la plantation d'eucalyptus tandis que les formes herbacées décroissent avec l'augmentation de l'âge. De même, le nombre de types biologiques varient avec l'âge de la plantation à eucalyptus. En suivant les trois espèces dominantes de cette forêt naturelle (Millettia laurentii et Markhamia tomentosa et Bosqueiopsis gillettii), on constate qu'elles possèdent plusieurs plantules dans le sous-bois des plantations d'eucalyptus, mais très peu de celles-ci n'arrivent au stage macrophanérophytes du fait du taux de mortalité élevé. Un schéma de reconstitution forestière a été proposé à partir des observations faites de cette forêt.

(C) 2016 International Formulae Group. All rights reserved.

Mots clés : Diversité floristique, dynamique de reconstitution, forêt naturelle, plantations à Eucalyptus, Brazzaville.

\section{Floristic diversity and reconstitution dynamics of the Zoological Park Forest under eucalyptus cultivation in Brazzaville, Congo}

\begin{abstract}
The Zoological Park forest is a natural relic's forest established at the center of Brazzaville's city. This ecosystem is undergoing anthropogenic pressure due to the growing needs in wood energy, wooden crafts and plants used in herbal medicine. The more degraded areas were reforested with eucalyptus. It was found in the
\end{abstract}


undergrowth of eucalyptus forests, the replenishment of natural forest. A diachronic study was made to follow this reconstruction. Plots of $600 \mathrm{~m}^{2}$ of different ages have been delineated in these plantations, in which all species of existing natural forest, DBH and the number of individuals were recorded. The results obtained show that morphological types of woody increase with age of the eucalyptus plantation while herbaceous forms decrease with increasing age. Similarly, the numbers of biological types vary with the age of the eucalyptus plantation. By following the three dominant species of this natural forest (Millettia laurentii) and Markhamia tomentosa and Bosqueiopsis gillettii, there they have several plants in the understory of eucalyptus plantations, but very few of these seedlings reach stage macrophanerophytes by virtue of the high mortality rate. A forest recovery scheme has been proposed from the observations of this forest.

(C) 2016 International Formulae Group. All rights reserved.

Keywords: Diversity floristic, dynamic of recovery, natural forest, plantations with Eucalyptus, Brazzaville.

\section{INTRODUCTION}

La gestion des écosystèmes forestiers intra urbains a toujours été un des problèmes majeurs des autorités municipales en Afrique en général et au Congo en particulier. La forêt de la Patte d'Oie, classée en 1938 comme réserve forestier est l'unique écosystème forestier intra urbain naturel de la ville de Brazzaville en République du Congo. Elle comptait, à sa création 240 ha, mais avec l'évolution démographique et l'extension de la ville de Brazzaville, elle est actuellement réduite en deux entités naturelles de moins de 92 ha. Il s'agit notamment de la forêt du parc zoologique et de la forêt de la cité des sciences. Ces deux entités sont continuellement exposées à des pressions anthropiques fortes pour des besoins de bois énergie et de construction et la présence des pistes piétonnes (Kimpouni et al., 2013). La forêt du parc zoologique qui a été estimée en 1952, date de la création du Parc Zoologique de Brazzaville, à 31,72 ha, ne comptait plus que de 25 ha en 1996 (Ikama Openga, 2013) et actuellement moins 12 ha compte tenu de la pression anthropique qu'elle subit. Cette évolution croissante du taux de déforestation risque d'entraîner l'érosion de certaines espèces sensibles (Tiokeng et al., 2015). Les deux îlots forestiers ont fait l'objet des études écologiques centrées essentiellement sur la régénération de la forêt naturelle, la dynamique et la dissémination des diaspores (Gakosso, 2009 et Kimpouni et al., 2013).
Les zones très dégradées de la forêt du Parc Zoologique ont été reboisées en Eucalyptus spp. Cependant, le soulignent Tassin et al. (2011), les plantations d'eucalyptus souffrent d'une image négative relative à leur diversité biologique du fait que généralement, dans le sous-bois de ces plantations, la régénération des espèces végétales locales ne se réalise pas du tout. Or, la biodiversité se révèle parfois plus élevée sous plantation d'eucalyptus qu'en d'autres espaces anthropisés voisins (exemple : terres agricoles ou de parcours, savanes herbeuses). Bien que les pressions sur les ressources naturelles ne cesse de s'accentuer, les données précises sur la dynamique de végétation et sur l'appauvrissement de la biodiversité font souvent défaut (Tankoano et al., 2015). C'est dans cette optique qu'il a été mis en évidence la diversité floristique et suivi la régénération de la forêt naturelle locale sous les plantations d'eucalyptus dans la forêt du parc zoologique de Brazzaville. Ce travail contribuera, comme le soulignent Baggnian et al. (2014) dans une étude similaire au Niger, d'une part, à fournir des éléments indicateurs des tendances majeures d'évolution qualitative et quantitative de la végétation naturelle sous plantations à eucalyptus. Plus spécifiquement nous avons entrepris de :

- identifier les espèces de forêts naturelles apparaissant sous les plantations d'eucalyptus ; 
- comprendre les mécanismes de recrutement des individus dans les divers types biologiques des arbres dominants de la forêt naturelle sous plantation d'eucalyptus ;

- dégager les différents groupes écologiques qui caractérisent la succession végétale naturelle ;

- comprendre les étapes et les mécanismes de reconstitution de la forêt.

\section{MATERIEL ET METHODES} Présentation du milieu d'étude

La forêt $\mathrm{du}$ Parc Zoologique de Brazzaville est contenue dans un triangle dont la base au Nord est limitée par l'avenue de la Patte d'Oie, le sommet au Sud, est limité par le carrefour du prolongement de la rue Dupleix et de l'avenue de l'aéroport Maya Maya, les côtés à l'Ouest par l'avenue de l'Aéroport Maya Maya et à l'Est par le prolongement de la rue Dupleix et la rue de la libération de Paris (Figure 1).

Sur le plan phytogéographique, la forêt du Parc Zoologique appartient au Domaine de Basse-Guinée et au Secteur de transition Congolo-Zambézienne du District de la Léfini (Descoings, 1975 ; Kimpouni et al., 1992) dont les forêts sont en majorité mésophile. La forêt naturelle du parc zoologique est une forêt à Millettia laurentii (Makany, 1976).

Le climat de Brazzaville est du type Bas Congolais (Samba-Kimbata, 1978) avec une pluviométrie moyenne annuelle variant entre 1300 et $1400 \mathrm{~mm}$, une saison sèche de 4 à 5 mois et les moyennes mensuelles de températures oscillant entre 23 et $25^{\circ} \mathrm{C}$. Les mois les plus chauds sont mars et avril; alors que juillet et août sont les plus frais (Vennetier, 1977; Adjanohoun et al., 1988). L'humidité relative est toujours supérieure à $70 \%$ avec un minimum relatif en février et en mars, alors que les minima oscillent entre 49 et $59 \%$. Les maxima restent au-dessus de $80 \%$ et varient entre 88 et 94\% (Makany, 1976). L'insolation moyenne mensuelle est comprise entre 120 et 160 heures, avec deux maxima en avril-mai et en novembre-décembre, et un minimum en juillet (Makany, 1976; Koechlin, 1961). Les vents, généralement faibles et de direction Ouest, Sud-Ouest et Nord-Ouest, dépassent rarement $5 \mathrm{~m} / \mathrm{s}$.

La géologie présente d'importantes séries essentiellement gréseuses et sableuses (Vennetier, 1977) : la série des plateaux Batéké et Bambio (grès silicifiés, sables) ; la série des cirques (argile sableuse) et la série du Stanley Pool et grès de Carnot constitués de grès et d'argilites. Les sols se développent sur des matériaux sableux, pauvres en base échangeables et très perméables. Ils sont ferrallitiques fortement désaturés et appauvris (Dénis, 1970). Brazzaville est arrosée par la Djiri au Nord et le Djoué au Sud (Vennetier, 1977). Outre ces affluents du fleuve Congo, on note la Tsiémé dans la zone nord, la Madoukou et la Mfoa dans la zone centrale qui se jettent dans le fleuve Congo. La M'filou à l'Ouest est un bras du Djoué. Le sol de la zone d'étude a une texture sablo-argileuse.

\section{Méthodes de collecte des données}

La dynamique de reconstitution de la forêt du Parc Zoologique a été réalisée à partir d'une étude diachronique en délimitant des placettes de $30 \mathrm{~m} \times 20 \mathrm{~m}$, soit $600 \mathrm{~m}^{2}$ sous les plantations d'eucalyptus d'âge différent. Les relevés floristiques ont été effectués dans des placettes des plantations à Eucalyptus spp de 2 ans (R1), de 4 ans (R2), de 7 ans (R3), de 12 ans (R4) et dans la forêt naturelle de plus de 12 ans (R5). Dans les relevés R2, R3, R4 et $\mathrm{R} 5$, il a été identifié toutes les espèces existantes, leur nombre d'individus, leur circonférence à $1,30 \mathrm{~m}$ du sol et les formes biologiques de chaque espèce. Pour le relevé $\mathrm{R} 1$, compte tenu de l'abondance des herbacées, une liste floristique a été établie sans compter le nombre d'individus par espèce. Cet échantillonnage systématique a été précédé d'une prospection botanique qui a permis d'identifier rapidement les arbres les 
plus abondants et dominants de la forêt naturelle.

\section{Méthodes d'interprétation des données Estimation de la diversité floristique}

La diversité floristique de l'écosystème a été estimée à partir des relevés floristiques qui ont permis de compter le nombre de familles et leur diversité spécifique.

\section{Types et spectres biologiques}

Les types biologiques identifiés sont liés à l'âge et à l'état phénologique des individus de chaque espèce rencontrée. Il a été utilisé le système de Raunkaier modifié par Lebrun (1947) et Guillaumet (1967). Le Tableau 1 ci-dessous présente les types biologiques rencontrés.

Les spectres brut et pondéré ont été calculés à partir des formules ci-dessous :

Spectre brut $(\%)=$ (nombre d'espèces par type biologique) $x$ 100/Nombre total d'espèces.

Spectre pondéré $(\%)=$ (nombre d'individus par type biologique) $\mathrm{x}$ 100/nombre total d'individus.

\section{RESULTATS}

\section{Evolution de la richesse floristique}

Il a été dénombré 145 espèces dans les cinq relevés floristiques effectués dans la forêt $\mathrm{du}$ Parc Zoologique. Ces espèces sont réparties dans 47 familles et 112 genres. Le Tableau 2 montre le nombre d'espèces recensées dans chaque relevé floristique.

Le calcul de l'indice de communauté de Jaccard, montre que les valeurs de cet indice sont supérieures à $21 \%$ (Tableau 3 ). Les relevés ayant des faibles écarts d'âges ont une valeur d'indice de Jaccard élevée et ont une composition floristique proche.

Par contre, plus l'écart d'âge est grand, moins l'indice de Jaccard est élevé et par conséquence, une composition floristique différente. Dans chaque relevé, sur la base de connaissance de chaque espèce recensée, il a noté son type morphologique ultime. La Figure 2 montre l'évolution des types morphologiques pendant le processus de reconstitution de la forêt du Parc Zoologique.

Il apparaît sur cette figure que les premières étapes de la reconstitution de la forêt sont colonisées par les herbacées et les plantules des lianes. A partir de 7 ans, le nombre d'espèces herbacées diminue et se stabilise tandis que les espèces arbustives et lianescentes deviennent de plus en plus en nombreuses.

\section{Evolution des spectres biologiques}

Les Figures 3 et 4 montrent l'évolution des spectres biologiques de la forêt du parc zoologique. Sur le plan floristique, le spectre brut montre que les nanophanérophytes sont abondamment représentés dans chaque étape de la reconstitution de la forêt (Figure 3). Ce fait prouve l'existence d'un stock de semences des espèces de forêt naturelle dans le sol des diverses plantations constituant ainsi un potentiel de régénération préexistante.

Cependant, sur le plan physionomique ou structural (Figure 4), on observe une distribution en cloche des nanophanérophytes.

En effet, la reconstitution de la forêt se fait en deux étapes. Une première étape de 2 à 7 ans, période pendant laquelle, il y a une restauration du potentiel ligneux de régénération. Une seconde étape à partir de 7 ans et plus, période pendant laquelle le recrutement des nanophanérophytes vers les méso et mégaphanérophytes commence à se réaliser effectivement. Les chaméphytes et les thérophytes majoritairement herbacés dans les étapes jeunes sont progressivement remplacés par les ligneux mieux adaptés aux nouvelles conditions du milieu induites par la structure de la forêt.

Pour estimer les pertes de recrutement des nanophanérophytes vers les micro, méso et mégaphanérophytes, trois populations des grands arbres de la forêt du Parc Zoologique 
ont été suivies en comptant le nombre d'individus dans chaque types biologiques dans les relevés R4 et R5 (Figure 5). En effet, le comportement des espèces végétales dans un milieu donné est sous la dépendance des facteurs environnementaux. Les types biologiques sont des formes d'adaptation aux conditions du milieu comme le souligne Adjanohoun et al. (1964).

La Figure 5 montre le suivi de l'évolution de la natalité réelle définie comme étant la germination des graines dans les conditions locales. Cette figure montre que quelle que soit la population considérée, la natalité réelle (nanophanérophytes + chaméphytes) reste élevée. Ce nombre de départ décroît considérablement au fur et à mesure de la croissance des plantules.

\section{Dynamique de la reconstitution de la forêt du Parc Zoologique}

En tenant compte de la composition floristique des différents relevés effectués des espèces de forêt naturelle sous les plantations d'Eucalyptus et des données de la littérature (Makany, 1976), la trajectoire de la reconstitution de la forêt du Parc Zoologique se présente de la manière suivante (Figure 6).

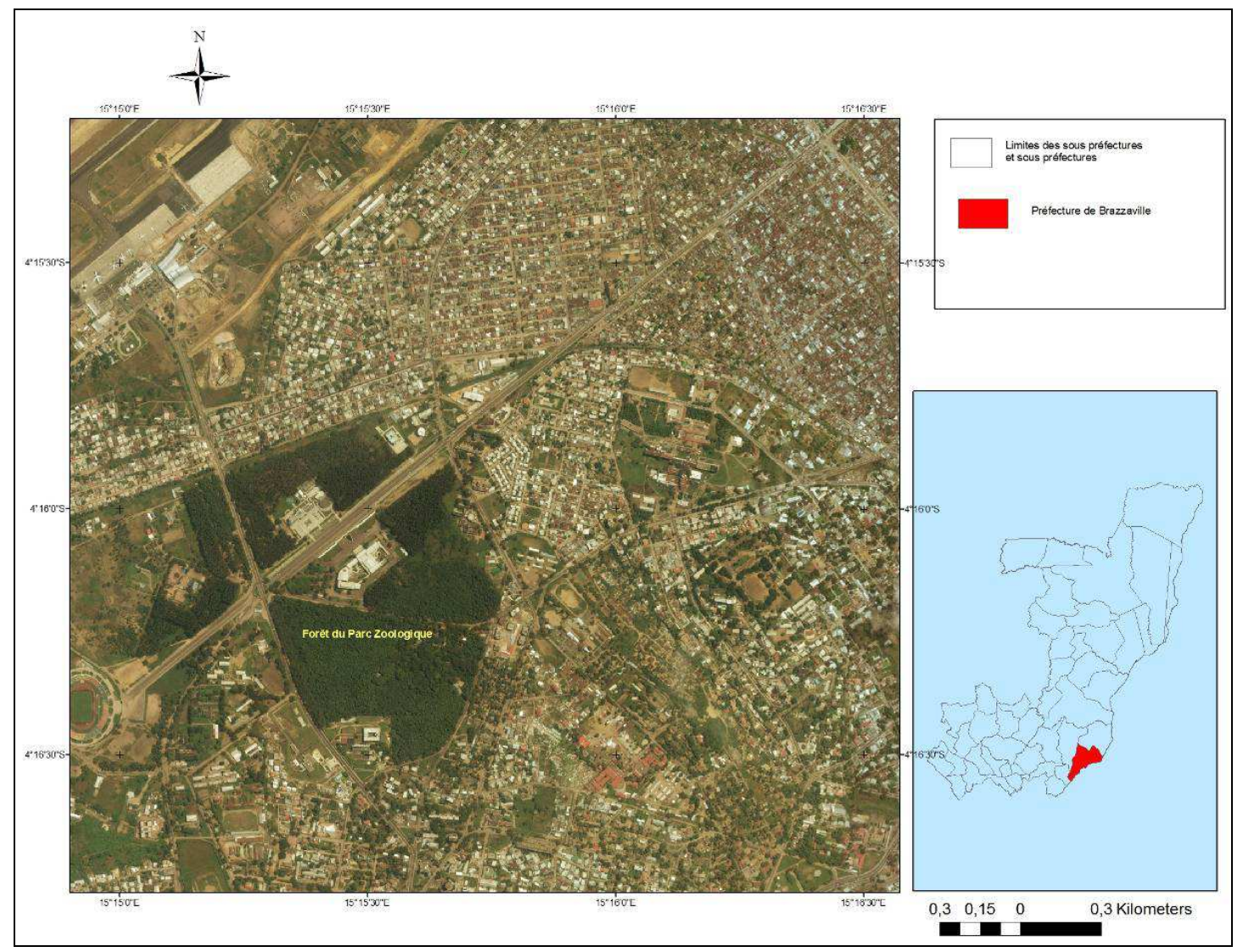

Figure 1: Situation géographique de la forêt du Parc Zoologique de Brazzaville. 


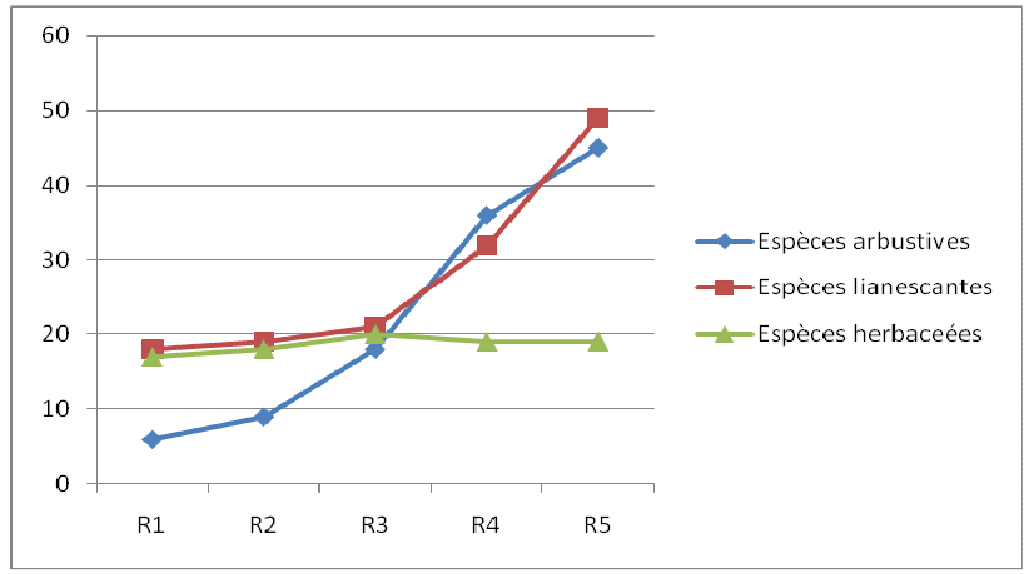

Figure 2 : Evolution des types morphologiques pendant le processus de la reconstitution forestière de la forêt du Parc Zoologique.

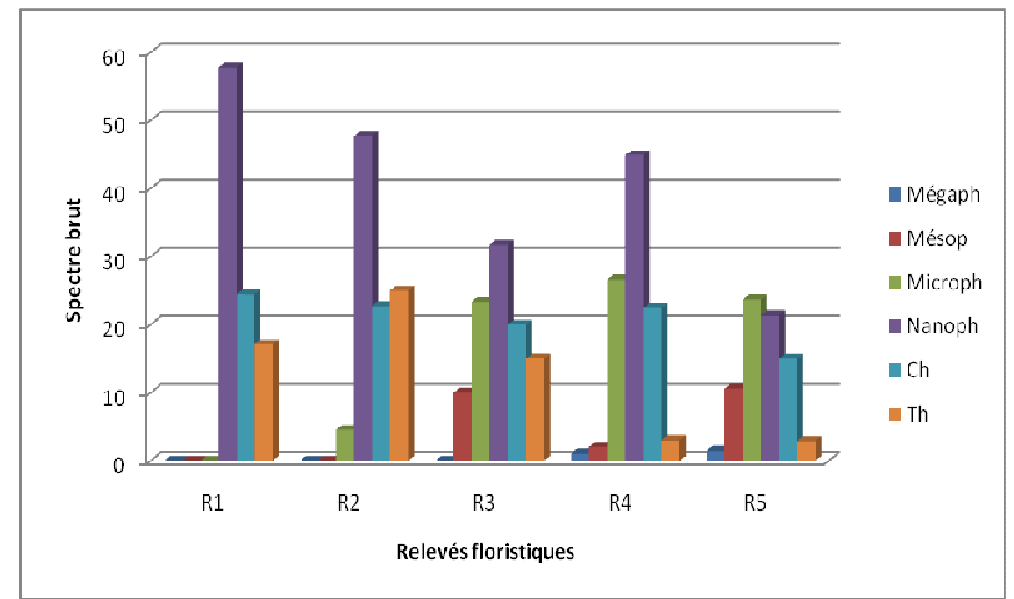

Figure 3 : Evolution du spectre brut pendant le processus de reconstitution de la forêt du Parc Zoologique.

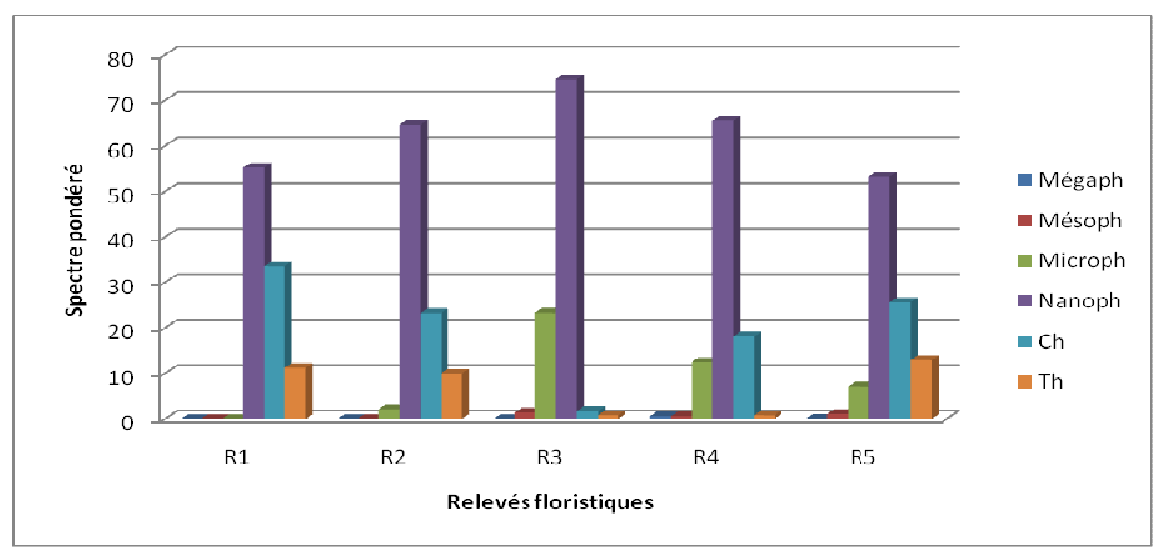

Figure 4 : Evolution du spectre pondéré pendant le processus de reconstitution de la forêt du Parc Zoologique. 


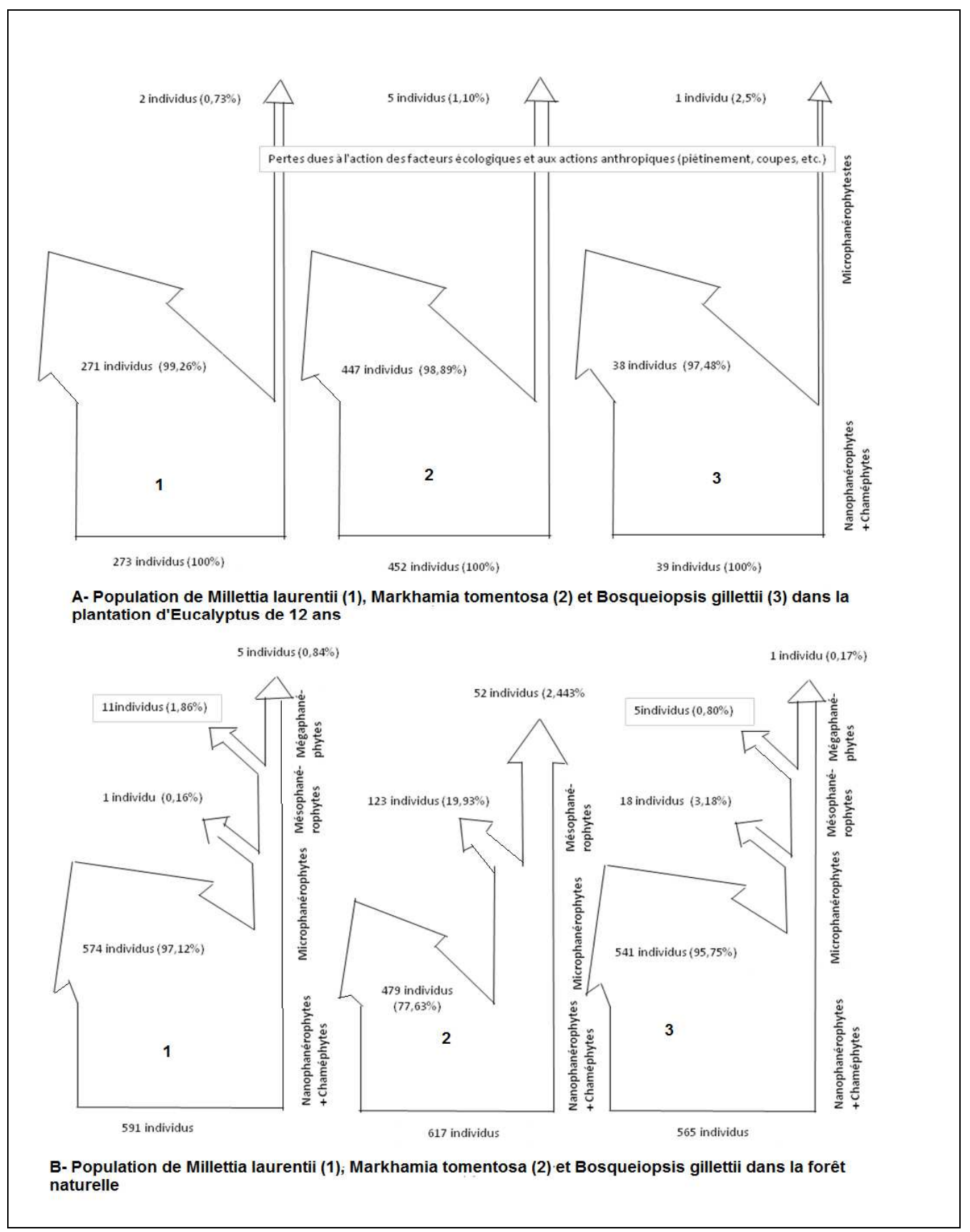

Figure 5 : Effets des facteurs environnementaux sur l'évolution des populations végétales pendant le processus de la régénération naturelle de la forêt du Parc Zoologique. 


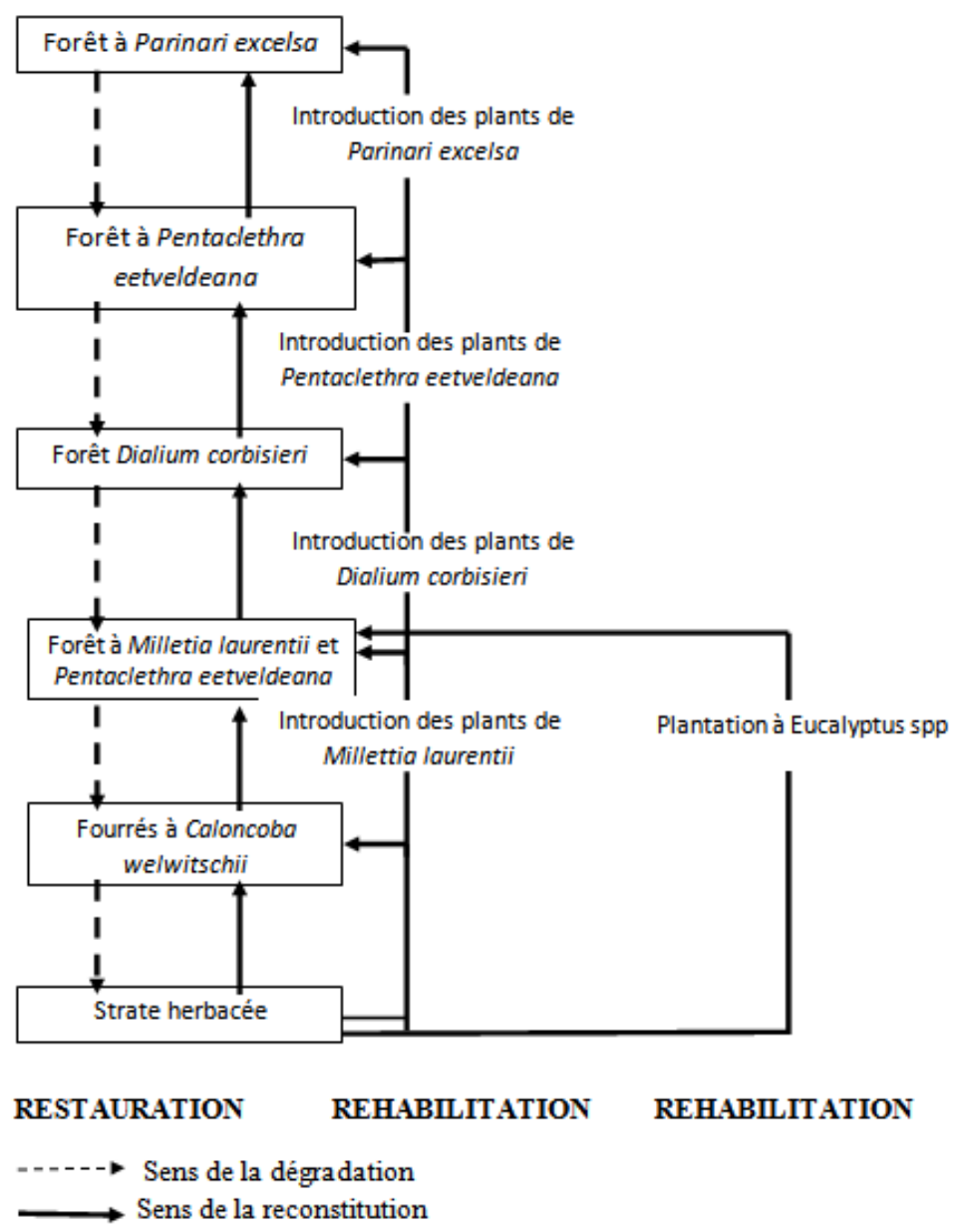

Figure 6: Processus de reconstitution forestière de la forêt du Parc Zoologique de Brazzaville.

Tableau 1 : Types biologiques utilisés pour la caractérisation des spectres biologiques.

\begin{tabular}{llcc}
\hline Types biologiques & & Abréviations & Dimensions \\
\hline & Mégaphanérophytes & Megaph & Hauteur supérieure à $30 \mathrm{~m}$ \\
& Mésophanérophytes & Mésoph & Hauteur comprise entre 10 et $30 \mathrm{~m}$ \\
Phanérophtes & Microphanérophytes & Microph & Hauteur comprise entre 2 et $10 \mathrm{~m}$ \\
& Nanophanérophytes & Nanoph & Hauteur comprise entre 1,25 et $2 \mathrm{~m}$ \\
\hline Chaméphytes & & $\mathrm{Ch}$ & Bourgeon terminal ne dépassant pas $1,25 \mathrm{~m}$ \\
Hémicryptophytes & & $\mathrm{Hc}$ & Plantes pérennes à bourgeons affleurant la \\
Thérophytes & & $\mathrm{Th}$ & surface du sol \\
Géophytes & & $\mathrm{Gr}$ & Plantes pérennes à bourgeons cachés dans le sol \\
\hline
\end{tabular}


Tableau 2: Répartition des taxons dans l'ensemble des relevés effectués sous plantations à eucalyptus dans la forêt du Parc Zoologique.

\begin{tabular}{llcccc}
\hline & Taxons & & $\begin{array}{c}\text { Nombre de } \\
\text { familles }\end{array}$ & $\begin{array}{c}\text { Nombre de } \\
\text { genres }\end{array}$ & $\begin{array}{c}\text { Nombre } \\
\text { d'espèces }\end{array}$ \\
\hline Phanérogames & Angiospermes & Monocots & 10 & 17 & 26 \\
& \multicolumn{2}{c}{$\begin{array}{c}\text { Dicots } \\
\text { Gymnospermes }\end{array}$} & 37 & 95 & 119 \\
& Total & 0 & 0 & 0 \\
& & 47 & 112 & 145 \\
\hline
\end{tabular}

Tableau 3 : Nombre d'espèces par relevé floristique.

\begin{tabular}{lcc}
\hline Relevés & âge & Nombre d'espèces \\
\hline R1 & 2 & 40 \\
R2 & 4 & 47 \\
R3 & 7 & 60 \\
R4 & 12 & 89 \\
R5 & Supérieur à 12 ans & 114 \\
\hline R1 = relevé $1 ;$ R2 = relevé $2 ; \mathrm{R} 3=$ relevé $3:$ R4 = relevé 4 et R5 = relevé 5
\end{tabular}

Tableau 4 : Valeurs de l'indice de communauté floristique de Jaccard.

\begin{tabular}{lccccc}
\hline Relevés & R1 & R2 & R3 & R4 & R5 \\
\hline R1 & 100 & 93,18 & 69,49 & 37,89 & 21,87 \\
R2 & 93,18 & 100 & 31,03 & 43,15 & 26,98 \\
R3 & 669,49 & 31,03 & 100 & 55,2 & 37 \\
R4 & 37,89 & 43,15 & 55,2 & 100 & 41,66 \\
R5 & 21,87 & 26,98 & 37 & 41,66 & 100 \\
\hline
\end{tabular}

\section{DISCUSSION}

\section{Evolution de la richesse floristique et de spectres biologiques}

Les résultats de ce travail ont montré que la richesse floristique et le nombre de spectres biologiques augmentent avec l'âge. Ces résultats confirment ceux obtenus par Koubouana et al. (2007) qui constatent que la variabilité du spectre biologique d'un écosystème décroît habituellement quand croît son niveau de dégradation. L'évolution en sens inverse du spectre brut des thérophytes par rapport à l'âge des stations d'inventaire laisse penser que ceux-ci sont relayés par les espèces sylvatiques plus adaptées aux nouvelles conditions du milieu. En effet, les thérophytes étant des espèces héliophiles, disparaissent en raison de la faible luminosité qui arrive dans le sous bois du fait de l'écran engendré par les couronnes des arbres. Cette diminution de l'éclairement favorise l'installation des espèces ligneuses forestières.

\section{Dynamique de la végétation}

La dynamique de la végétation est sous la dépendance des facteurs environnementaux (facteurs écologiques et facteurs anthropiques). En effet? la réduction du nombre d'individus du stade plantule vers le stade grand arbre s'explique par l'influence des facteurs écologiques et anthropiques qui sont à l'origine des mortalités massives observées dans les différentes populations végétales. L'action des facteurs écologiques est prépondérante dans les premiers stades de la régénération et, elle se traduit par : 
- la compétition intra et interspécifique entre les différentes espèces (compétition pour la lumière et nutrition hydrique et minérale) ;

- les phénomènes de prédation et de parasitisme (insectes phytophages, champignons parasites des feuilles, etc.).

Dans la forêt du Parc Zoologique, l'action des facteurs environnementaux est renforcée par les perturbations anthropiques qui se traduisent par (Kimpouni et al., 2013) :

- le piétinement des jeunes individus et l'apport des déchets ménagers (sacs plastiques, boîtes de conserve) observés dans la forêt ;

- les coupes abusives d'abres, non autorisées.

En ce qui concerne la dynamique de reconstitution de cette forêt, Koubouana et al. (2007) ONT montré que le climax des forêts sur sols sablo-argileux des Plateaux Téké serait la forêt à Parinari excelsa et que la trajectoire suivie par les zones dégradées pour atteindre ce climax, passe par des états alternatifs stables. Ces états alternatifs stables sont représentés par la forêt à Dialium corbisieri, la forêt à Pentaclethra eetveldeana et à Millettia laurentii. De ce fait, la forêt du Parc Zoologique ayant comme arbres dominants Millettia laurentii n'est qu'un état alternatif stable de la reconstitution de la forêt climatique à Parinari excelsa.

La forêt actuelle du parc Zoologique, qui est une forêt à Millettia laurentii ne serait qu'un état alternatif stable de l'évolution progressive vers le climax. Le maintien de cette forêt à cet état s'explique par l'action des facteurs environnmentaux et l'action de l'homme.

\section{Conclusion}

Ce travail a montré que dans les sous bois des plantations à Eucalyptus, apparaissent les espèces de la forêt naturelleles. Au total, 145 espèces ont été inventoriées dans les cinq relevés floristiques effectués dans la forêt du Parc Zoologique. Ces espèces sont réparties dans 47 familles et 112 genres.
Le calcul de l'indice de communauté de Jaccard a donné des valeurs inférieures à 50\%, ce qui montre que les cinq relevés floristiques effectués sont différents sur le plan composition floristique.

En effet, pendant le processus de reconstitution de la forêt naturelle, il a été noté une augmentation des types biologiques en fonction de l'âge des différents relevés effectués.

Pendant les premières étapes de la régénération de la forêt du Parc Zoologique quelle que soit la trajectoire que l'on peut utiliser pour réhabilitr la forêt, les espèces sylvatiques prennent toujours le dessus sur les espèces savanicoles. Ce qui permet de penser que le climat de Brazzaville est propice au développement de la forêt. Malheureusement, cette forêt subit une forte pression anthropique ce qui a pour conséquence une diminution du nombre d'espèces et de spectres biologiques dans les zones dégradées et un blockage de l'évolution progressive de l'écosystème forestier. Pour faciliter la reprise de l'évolution progressive de la forêt, trois activités ont été menées :

- la restauration qui est un processus qui permet à la forêt de se reconstituer toute seule sans intervention de l'homme. Pour que la restauration ait lieu, il faut que la forêt soit exemptée de toute pression anthropique supplémentaire.

- la réhabilitation, quant à elle, permet de corriger et d'accélérer le processus de régénération de la forêt. Dans cette activité, comme pour la réaffectation, c'est l'homme qui intervient en regarnissant la forêt par introduction des plants des espèces dominantes.

- la réaffectation est une activité de l'homme aucours de laquelle la trajectoire de la régénération est totalement détournée. Elle est utilisée dans les zones dégradées en mettant en plantantion des espèces à croissance rapide qui créent des conditions nécessaires à la germination des diaspores des espèces autochtones en dormance dans le sol. C'est le cas des parties de la forêt du Parc 
Zoologique étudiée, représentées par les plantations d'Eucalyptus dans lesquelles réapparaissent dans le sous bois les germinations des espèces autochtones.

\section{REFERENCES}

Adjanohoun E. 1964 : Végétation des savanes et des rochers découverts en Côte d'Ivoire Centrale, Mémoire ${ }^{\circ}$ 7, ORSTOM, Paris, $178 \mathrm{p}$.

Adjanohoun EJ, Ahyi AMR, Ake Asi L, Baniakina J, Chibon P, Cusset G, Doulou $\mathrm{V}$, Enzanza A, Eyeme J, Goudote E, Keita E, Mbemba C, Mollet J, Moutsamboté JM, Mpati J, Sita P. 1988. Contribution aux Etudes Ethnobotaniques et Floristiques en République Populaire du Congo : Médecine Traditionnelle et Pharmacopée. ACCT: Paris ; 302p.

Baggnian I, Adam T, Adamou MM, Chaibou I, Mahamane A. 2014. Structure et dynamique de la végétation ligneuse juvénil issue de la régénération naturelle assistée (RNA) dans le Centre-Sud du Niger. Int. J. Biol. Chem. Sci., 8(2): 649665.

Denis B. 1970. Les Sols de la Région de Brazzaville. ORSTOM: Brazzaville.

Descoings B. 1975. Les grandes régions naturelles du Congo. Candollea, 30: 91120.

Gakosso G. 2009. Diversité floristique et potentialité régénérative des îlots forestiers naturels de la Patte d'Oie. Mémoire de CAPES, ENS, UMNG, Brazzaville, p. 68.

Ikama Openga C. 2013. Dynamique de la perte du couvert forestier de la réserve forestière de la Patte d'Oie (Brazzaville, pour la période de 1946 à 2014). Mem.
Master, Univ. Marirn Ngouabi, Brazzaville, Congo, 57p.

Lebrun J. 1947. La Végétation de la Pleine Alluviale au Sud Lac Edouard. Bruxelles, (vol. 22). Inst. Parc Nat: Congo-Belge ; $800 \mathrm{p}$.

Kimpouni V, Lejoly J, Lisowski S. 1992. Les Eriocaulaceae $d u$ Congo. Fragmenta Floristica Geobotanica, 37: 127-145.

Kimpouni V, Mbou P, Gakosso G, Mtom M. 2013. Biodiversité floristique du sousbois et régénération naturelle de la forêt de la Patte d'Oie de Brazzaville, Congo. Int. J. Biol Chem. Sci., 7(3): 1255-1270.

Koubouana F, Ngoliélé A, Nsongola G. 2007. Evolution des paramètres floristiques pendant la régénération des forêtsde la réserve de la Léfini (Congo-Brazzaville). Ann. Univ. Marien Ngouabi, 8(4): 10-21.

Makany L. 1976. Végétation des Plateaux Téké (Congo). Collection des travaux de l'Univ. De Brazzaville, 301p.

Samba-Kimbata MJ. 1978. Le climat du basCongo. Thèse 3è cycle Géographie, Univ. De Dijon, 280p.

Tankoano B, Hien M, DIBI HN. 2015. Dynamique spatio-temporelle des savanes de la forêt classée de Tiogo au Burkina Faso. Int. J. Biol Chem. Sci., 9(4): 19832000.

Tassin J, Missamba Lola P, Marien JN. 2011. Biodiversité des plantations d'eucalyptus. BFT., 309(3): 27-35.

Tioheng B, Mapongmetsem P-M, Nguetsop VF, Tacham W. 2015. Biodiversité floristique et regénération naturelles $\mathrm{s}$ deLebaur les Hautes Terres de Lebialem (Ouest Cameroun). Int. J. Biol. Chem. Sci., 9(1): 56-68.

Vennetier P. 1977. Atlas du Congo ; Ed. Jeune Afrique ; $32 \mathrm{p}$. 